LPC 9653

INFNCA-TH9620

hep-ph/9611337

November 1996

\title{
Production of meson pairs, involving tensor and pseudotensor mesons, in photon-photon collisions
}

\author{
Ladonne Houra-Yaou, Paul Kessler, and Joseph Parisi \\ Laboratoire de Physique Corpusculaire, Collège de France \\ 11, Place Marcelin Berthelot, F-75231 Paris Cedex 05, France \\ Francesco Murgia \\ Istituto Nazionale di Fisica Nucleare, Sezione di Cagliari \\ Via Ada Negri 18, I-09127 Cagliari, Italy \\ Johan Hansson \\ Department of Physics, Luleå University of Technology, S-95187 Luleå, Sweden
}

\begin{abstract}
Starting from a bound-state model of weakly bound quarks for $(q \bar{q})$ mesons, we derive a formalism for computing the production or decay of such mesons, whatever the value of their internal orbital angular momentum $L$. That approach appears as a natural generalization of the Brodsky-Lepage formalism (valid only for $L=0$ ) that has been widely used in recent years for the computation of exclusive processes in perturbative QCD. We here apply it to the production, in photon-photon collisions, of : $i$ ) tensor-meson pairs ; ii) pseudotensor-meson pairs ; iii) hybrid pairs made of a pion and a pseudotensor meson. The numerical results we obtain allow for some hope of experimentally identifying such pairs, in the charged channels, at high-energy $e^{+} e^{-}$colliders of the next generation, provided integrated luminosities as high as $\approx 10^{40} \mathrm{~cm}^{-2}$ can be reached.
\end{abstract}




\section{Introduction}

Some fifteen years ago Brodsky and Lepage presented a formalism, based on perturbative quantum chromodynamics, that allows for the calculation of exclusive processes involving particle production or decay [1]. Over the years it has given rise to a number of more or less successful applications, such as for instance hadron pair production in photon-photon collisions [2]. However it is well known that, as far as mesons are concerned, the BrodskyLepage formalism can only be applied to $(q \bar{q})$ states with zero orbital angular momentum, i.e. to pseudoscalar and vector $(q \bar{q})$ mesons. In particular, the production or decay of scalar, axial-vector or tensor $(q \bar{q})$ mesons, which all have their orbital angular momentum $L$ equal to one, as well as of pseudotensor $(q \bar{q})$ mesons with $L=2$, cannot be treated in that formalism.

The purpose of this paper is to define a procedure for the calculation of exclusive processes involving the production or decay of $(q \bar{q})$ mesons with any orbital angular momentum $L$. Starting from a bound-state model of weakly bound quarks for such mesons [3], we shall derive an approach that will basically appear as a natural generalization of the Brodsky-Lepage formalism. Actually the formalism here shown is closely related to the one presented by some of the authors in former papers bearing on the calculation of processes that involve the production or decay of $(g g)$ glueballs [4, 5, 6, 7]. Let us also recall the mathematical framework that was developed in a similar context by Benayoun and Froissart [8].

Section 2 of this paper will be devoted to the derivation of our formalism. In section 3 that formalism will be applied to the production, in photon-photon collisions, of : (i) $(q \bar{q})$ tensor-meson pairs ; (ii) $(q \bar{q})$ pseudotensor-meson pairs ; (iii) hybrid pairs made of a pion and a $(q \bar{q})$ pseudotensor meson ; numerical results will be presented for those three applications. Section 4 contains a brief conclusion. Details of calculation are given in three Appendices.

\section{A formalism for the production of $(q \bar{q})$ mesons with any orbital angular momentum $L$}

Let us consider a production process $a b \rightarrow Q c$, where $Q$ is a $(q \bar{q})$ meson and $a, b, c$ are any particles. This process is represented schematically in Fig. 1, both in its center-of-mass frame (A) and in the meson rest frame (B). In the latter the quark $q$ resp. the antiquark $\bar{q}$ is moving inside the meson with a Fermi momentum $\mathbf{k}$ resp. $\mathbf{- k}$. Let us notice from the start that the demonstration presented hereafter can be extended in a trivial way to the inverse process $Q c \rightarrow a b$.

Calling $\mathcal{M}$ the amplitude of the process $a b \rightarrow Q c$ and $\mathcal{T}$ that of the corresponding process at the parton level, i.e. $a b \rightarrow q \bar{q} c$, a prescription given in Ref. [3] relates $\mathcal{M}$ and $\mathcal{T}$ in the following way :

${ }^{1}$ With respect to Eq. (2.1) of Ref. [3] we here introduce a slight modification (replacing the quark and antiquark masses by the corresponding energies in the meson rest frame) which has no consequence from the point of view of practical applications of the formalism here presented. 


$$
\mathcal{M}=\int d^{3} \mathbf{k} \Psi^{*}(\mathbf{k}) \mathcal{T} \frac{(2 \pi)^{3 / 2}(2 M)^{1 / 2}}{(2 \pi)^{3 / 2} \sqrt{2\left(m^{2}+\mathbf{k}^{2}\right)^{1 / 2}}(2 \pi)^{3 / 2} \sqrt{2\left(\bar{m}^{2}+\mathbf{k}^{2}\right)^{1 / 2}}},
$$

where $\Psi(\mathbf{k})$ is the Fermi-momentum distribution of the quark within the meson (or, in other words, the meson wave function in momentum space, defined in the meson rest frame). The last factor at right hand arises from the normalization of the S matrix. $M, m$ and $\bar{m}$ are, respectively, the masses of the meson, the quark and the antiquark.

In the spirit of the Brodsky-Lepage formalism (see also Ref. [8]), we define the fourvectors $q^{\mu}$ and $\bar{q}^{\mu}$ of the quark and antiquark, respectively, as follows :

$$
q^{\mu}=x Q^{\mu}+k^{\mu} \quad \bar{q}^{\mu}=(1-x) Q^{\mu}-k^{\mu},
$$

where $Q^{\mu}$ is the meson four-momentum, and $k^{\mu}$ is the four-dimensional generalization of the above-mentioned Fermi momentum (in the meson rest frame its energy and momentum components are : $0, \mathbf{k}$ ). The energy of the quark resp. antiquark in the meson rest frame, as derived from Eq. (2), is given by :

$$
q_{0}=\sqrt{m^{2}+\mathbf{k}^{2}}=x M \quad \bar{q}_{0}=\sqrt{\bar{m}^{2}+\mathbf{k}^{2}}=(1-x) M .
$$

Eq. (11) is thus generalized, in the spirit of Brodsky-Lepage, into :

$$
\mathcal{M}=\frac{1}{\sqrt{2 M}} \int \frac{d^{3} \mathbf{k}}{(2 \pi)^{3 / 2}} \Psi^{*}(\mathbf{k}) \int \frac{\Phi_{N}^{*}(x) d x}{\sqrt{x(1-x)}} \mathcal{T}
$$

where $\Phi_{N}(x)$ is the $(q \bar{q})$ meson's distribution amplitude, normalized so that $\int \Phi_{N}(x) d x=1$.

To be precise, we notice that the amplitude $\mathcal{M}$ is a function of $E$ (the total c.m. energy of the process) and of the scattering angle $\Theta$ [see Fig. 1(A)], while $\mathcal{T}$ is a function of $E, \Theta, k(\equiv|\mathbf{k}|)$ and of the angles $\theta$ and $\phi$ [see Fig. 1(B)] as well as of $x$. On the other hand $\mathcal{M}$ depends on the following quantum numbers : $L$ (the meson's internal orbital angular momentum), $S$ (its intrinsic spin), $\Lambda_{L}$ (the component of $L$ on the $z$-axis of Fig. 1), $\Lambda_{S}$ (the component of $S$ on that axis), and in addition on the helicities $\lambda_{a}, \lambda_{b}, \lambda_{c}$ of particles $a, b, c$ respectively ; $\mathcal{T}$ depends on $S, \Lambda_{S}$ and $\lambda_{a}, \lambda_{b}, \lambda_{c}$.

We then apply the relation :

$$
\mathcal{M}_{\lambda_{a} \lambda_{b} \lambda_{c}}^{L S J \Lambda}(E, \Theta)=\sum_{\Lambda_{L}}\left\langle L S \Lambda_{L} \Lambda_{S} \mid L S J \Lambda\right\rangle \mathcal{M}_{\lambda_{a} \lambda_{b} \lambda_{c}}^{L S \Lambda_{L} \Lambda_{S}}(E, \Theta)
$$

where $J$ is the meson's total spin and $\Lambda\left(=\Lambda_{L}+\Lambda_{S}\right)$ the corresponding component on the $z$-axis, and where we use the conventional notation $\left\langle j_{1} j_{2} m_{1} m_{2} \mid j_{1} j_{2} j m\right\rangle$ for ClebschGordan coefficients ; on the other hand we factorize the meson wave function into a radial and an angular part, as follows : 


$$
\Psi(\mathbf{k})=R_{L}(k) Y_{L \Lambda_{L}}(\theta, \phi),
$$

and finally, using the well-known expression of the spherical harmonics :

$$
Y_{L \Lambda_{L}}(\theta, \phi)=\sqrt{\frac{2 L+1}{4 \pi}} d_{\Lambda_{L} 0}^{L}(\theta) e^{i \Lambda_{L} \phi}
$$

where $d_{\Lambda_{L} 0}^{L}(\theta)$ is a Wigner rotation matrix element, Eq. (幽 becomes :

$$
\begin{aligned}
\mathcal{M}_{\lambda_{a} \lambda_{b} \lambda_{c}}^{L S J \Lambda}(E, \Theta) & =\frac{\sqrt{2(2 L+1) \pi}}{\sqrt{M}} \int \frac{k^{2} d k}{(2 \pi)^{3 / 2}} R_{L}^{*}(k) \int \frac{d(\cos \theta)}{2} \int \frac{d \phi}{2 \pi} \\
& \times \sum_{\Lambda_{L}} \zeta^{L S J \Lambda_{L} \Lambda_{S}}(\theta, \phi) \int \frac{\Phi_{N}^{*}(x) d x}{\sqrt{x(1-x)}} \mathcal{T}_{\lambda_{a} \lambda_{b} \lambda_{c}}^{S \Lambda_{S}}(E, \Theta, k, \theta, \phi, x),
\end{aligned}
$$

where we define :

$$
\zeta^{L S J \Lambda_{L} \Lambda_{S}}(\theta, \phi)=\left\langle L S \Lambda_{L} \Lambda_{S} \mid L S J \Lambda\right\rangle d_{\Lambda_{L} 0}^{L}(\theta) e^{-i \Lambda_{L} \phi}
$$

Let us now assume that, in the c.m. frame of the reaction $a b \rightarrow Q c$, the meson is extreme-relativistic, i.e. $M \ll E$; in other words, the c.m. frame is to be considered an "infinite-momentum frame". It is then easily shown (see Appendix A) that $\mathcal{T}$ becomes independent of $\phi$, so that, because of the exponential in Eq. (9), only contributions with $\Lambda_{L}=0$ (and consequently $\Lambda=\Lambda_{S}$ ) remain finite f. Eq. (8) is thus simplified into :

$$
\begin{aligned}
\mathcal{M}_{\lambda_{a} \lambda_{b} \lambda_{c}}^{L S J \Lambda}(E, \Theta) & =\frac{\sqrt{2(2 L+1) \pi}}{\sqrt{M}} \int \frac{k^{2} d k}{(2 \pi)^{3 / 2}} R_{L}^{*}(k) \int \frac{d(\cos \theta)}{2} \\
& \times \zeta^{L S J \Lambda}(\theta) \int \frac{\Phi_{N}^{*}(x) d x}{\sqrt{x(1-x)}} \mathcal{T}_{\lambda_{a} \lambda_{b} \lambda_{c}}^{S \Lambda}(E, \Theta, k, \theta, x),
\end{aligned}
$$

where

$$
\zeta^{L S J \Lambda}(\theta)=\langle L S 0 \Lambda \mid L S J \Lambda\rangle d_{00}^{L}(\theta)
$$

At this point we notice that expanding the integral over $x$ and $\theta$, in the r.h. member of Eq. (10), in increasing powers of $k$, we expect the resulting power series to start with the term in $k^{L}$, i.e. :

${ }^{2} \Lambda$ is thus limited to the values $0, \pm 1$. It results (as already noticed in Ref. [8]) that, when a prompt meson is found in a helicity state \pm 2 in a high-energy reaction, there is a good chance that it might be a glueball. 


$$
\begin{gathered}
\int \frac{d(\cos \theta)}{2} \zeta^{L S \Lambda}(\theta) \int \frac{\Phi_{N}^{*}(x) d x}{\sqrt{x(1-x)}} \mathcal{T}_{\lambda_{a} \lambda_{b} \lambda_{c}}^{S \Lambda}(E, \Theta, k, \theta, x) \\
=a_{L} k^{L}+a_{L+1} k^{L+1}+a_{L+2} k^{L+2}+\ldots
\end{gathered}
$$

We now assume, as in [3], that in the meson rest frame the quarks are essentially nonrelativistic, i.e. their Fermi-momentum distribution is sharply peaked towards $k \rightarrow 0$, so that in the r.h. member of Eq. (12) only the lowest-order term is to be retained. We then set :

$$
\int \frac{k^{2} d k}{(2 \pi)^{3 / 2}} k^{L} R_{L}(k)=C_{L} .
$$

As has been shown in Refs. [3, 田], this parameter $C_{L}$ can be connected as follows with the meson wave function at the origin in configuration space :

$$
C_{L}=\frac{(-i)^{L}(2 L+1) ! !}{4 \pi L !}\left[\frac{d^{L}}{d r^{L}} R_{L}(r)\right]_{r \rightarrow 0} .
$$

Replacing the variable $k$ by the dimensionless one $\beta=2 k / M$, and setting

$$
f_{L}=\left(\frac{2}{M}\right)^{L+1 / 2} \sqrt{(2 L+1) \pi} C_{L}
$$

we now rewrite Eq. (10) as follows :

$$
\mathcal{M}_{\lambda_{a} \lambda_{b} \lambda_{c}}^{L S J \Lambda}(E, \Theta)=f_{L}^{*} \lim _{\beta \rightarrow 0} \frac{1}{\beta^{L}} \int \frac{d(\cos \theta)}{2} \zeta^{L S J \Lambda}(\theta) \int \frac{\Phi_{N}^{*}(x) d x}{\sqrt{x(1-x)}} \mathcal{T}_{\lambda_{a} \lambda_{b} \lambda_{c}}^{S \Lambda}(E, \Theta, \beta, \theta, x)
$$

It can easily be checked that, for $L=0$, Eq. (16) leads exactly to the same expression as provided by the Brodsky-Lepage formalism, defining the distribution amplitude $\Phi(x)$ used by those authors as : $\Phi(x)=f_{0} \Phi_{N}(x)$. Using the charged meson's leptonic decay for normalization, one gets : $\left|f_{0}\right|=f_{M} /(2 \sqrt{3})$, where $f_{M}$ is the meson's leptonic decay constant; in particular, $f_{\pi} \cong 93 \mathrm{MeV}$.

Eq. (16) is easily generalized for the case of $(q \bar{q})$ meson pair production, i.e. $a b \rightarrow Q Q^{\prime}$; one gets

$$
\begin{aligned}
\mathcal{M}_{\lambda_{a} \lambda_{b}}^{L S J \Lambda, L^{\prime} S^{\prime} J^{\prime} \Lambda^{\prime}}(E, \Theta) & =f_{L}^{*} f_{L}^{\prime *} \lim _{\beta, \beta^{\prime} \rightarrow 0} \frac{1}{\beta^{L} \beta^{\prime L^{\prime}}} \int \frac{d(\cos \theta)}{2} \zeta^{L S J \Lambda}(\theta) \int \frac{d\left(\cos \theta^{\prime}\right)}{2} \zeta^{L^{\prime} S^{\prime} J^{\prime} \Lambda^{\prime}}\left(\theta^{\prime}\right) \\
& \times \int \frac{\Phi_{N}^{*}(x) d x}{\sqrt{x(1-x)}} \int \frac{\Phi_{N}^{\prime *}\left(x^{\prime}\right) d x^{\prime}}{\sqrt{x^{\prime}\left(1-x^{\prime}\right)}} \mathcal{T}_{\lambda_{a} \lambda_{b}}^{S \Lambda, S^{\prime} \Lambda^{\prime}}\left(E, \Theta, \beta, \beta^{\prime}, \theta, \theta^{\prime}, x, x^{\prime}\right),
\end{aligned}
$$


where $\mathcal{M}$ refers to the process $a b \rightarrow Q Q^{\prime}$, while $\mathcal{T}$ refers to $a b \rightarrow q \bar{q} q^{\prime} \bar{q}^{\prime}$ and where all symbols without and with "prime" are pertaining to $Q$ and $Q^{\prime}$ respectively.

The expressions of momentum four-vectors and projectors of spinor pairs, to be used in the calculation of the amplitudes $\mathcal{T}$, both for $a b \rightarrow Q c$ and $a b \rightarrow Q Q^{\prime}$, are shown in Appendix A.

\section{Application to the production of tensor and pseu- dotensor mesons in photon-photon collisions}

We shall here consider the following processes :

(i) Pair production, in $\gamma \gamma$ collisions, of $(q \bar{q})$ tensor mesons, i.e. of $f_{2}(1270), a_{2}(1310)$ and $f_{2}^{\prime}(1525)$, all of them with quantum numbers $L=S=1, J=2$.

(ii) Pair production, in $\gamma \gamma$ collisions, of $(q \bar{q})$ pseudotensor mesons $\pi_{2}(1670)$ with $L=2$, $S=0, J=2$.

(iii) Production, in $\gamma \gamma$ collisions, of hybrid pairs made of one pion $(L=S=J=0)$ and one $\pi_{2}$ meson $(L=2, S=0, J=2)$.

For those three types of reactions, Eq. (17) becomes respectively :

$$
\begin{aligned}
\mathcal{M}_{\lambda_{\gamma} \lambda_{\gamma}^{\prime}}^{112 \Lambda, 112 \Lambda^{\prime}}(E, \Theta) & =f_{1}^{*} f_{1}^{\prime *} \lim _{\beta, \beta^{\prime} \rightarrow 0} \frac{1}{\beta \beta^{\prime}} \int \frac{d(\cos \theta)}{2} \zeta^{112 \Lambda}(\theta) \int \frac{d\left(\cos \theta^{\prime}\right)}{2} \zeta^{112 \Lambda^{\prime}}\left(\theta^{\prime}\right) \\
& \times \int \frac{\Phi_{N}^{*}(x) d x}{\sqrt{x(1-x)}} \int \frac{\Phi_{N}^{\prime *}\left(x^{\prime}\right) d x^{\prime}}{\sqrt{x^{\prime}\left(1-x^{\prime}\right)}} \mathcal{T}_{\lambda_{\gamma} \lambda_{\gamma}^{\prime}}^{1 \Lambda, 1 \Lambda^{\prime}}\left(E, \Theta, \beta, \beta^{\prime}, \theta, \theta^{\prime}, x, x^{\prime}\right) \\
\mathcal{M}_{\lambda_{\gamma} \lambda_{\gamma}^{\prime}}^{2020,2020}(E, \Theta)= & f_{2}^{*} f_{2}^{\prime *} \lim _{\beta, \beta^{\prime} \rightarrow 0} \frac{1}{\beta^{2} \beta^{\prime 2}} \int \frac{d(\cos \theta)}{2} \zeta^{2020}(\theta) \int \frac{d\left(\cos \theta^{\prime}\right)}{2} \zeta^{2020}\left(\theta^{\prime}\right) \\
\times & \int \frac{\Phi_{N}^{*}(x) d x}{\sqrt{x(1-x)}} \int \frac{\Phi_{N}^{\prime *}\left(x^{\prime}\right) d x^{\prime}}{\sqrt{x^{\prime}\left(1-x^{\prime}\right)}} \mathcal{T}_{\lambda_{\gamma} \lambda_{\gamma}^{\prime}}^{00,00}\left(E, \Theta, \beta, \beta^{\prime}, \theta, \theta^{\prime}, x, x^{\prime}\right) \\
\mathcal{M}_{\lambda_{\gamma} \lambda_{\gamma}^{\prime}}^{0000,2020}(E, \Theta) & =f_{0}^{*} f_{2}^{\prime *} \lim _{\beta^{\prime} \rightarrow 0} \frac{1}{\beta^{\prime 2}} \int \frac{d\left(\cos \theta^{\prime}\right)}{2} \zeta^{2020}\left(\theta^{\prime}\right) \\
& \times \int \frac{\Phi_{N}^{*}(x) d x}{\sqrt{x(1-x)}} \int \frac{\Phi_{N}^{\prime *}\left(x^{\prime}\right) d x^{\prime}}{\sqrt{x^{\prime}\left(1-x^{\prime}\right)}} \mathcal{T}_{\lambda_{\gamma} \lambda_{\gamma}^{\prime}}^{00,00}\left(E, \Theta, \beta^{\prime}, \theta^{\prime}, x, x^{\prime}\right)
\end{aligned}
$$

In writing down Eq. (20) we have made use of the fact that, for $\beta \rightarrow 0$, the amplitude

$\mathcal{T}_{\lambda_{\gamma} \lambda_{\gamma}^{\prime}}^{00,00}$ here becomes independent of $\theta$ (as can easily be checked), and that in addition one has : $\int[d(\cos \theta) / 2] \zeta^{0000}(\theta)=1$. 
In order to use Eqs. (18)-(20) for the evaluation of the amplitudes at the hadron level, we must choose the distribution amplitudes $\Phi_{N}(x), \Phi_{N}^{\prime}\left(x^{\prime}\right)$ and, on the other hand, determine the values of the constants $f_{L}, f_{L^{\prime}}^{\prime}$ involved.

For the distribution amplitudes (DA) of the tensor and pseudotensor mesons, we shall make two different choices :

(i) The so-called nonrelativistic DA [1] :

$$
\Phi_{N}(x)=\delta\left(x-\frac{1}{2}\right)
$$

(ii) A generalization of the so-called asymptotic DA [1], namely :

$$
\Phi_{N}(x)=\frac{2^{2 L+3} \Gamma(L+5 / 2)}{\sqrt{\pi} \Gamma(L+2)} x^{L+1}(1-x)^{L+1},
$$

where $\Gamma(z)$ is the well-known Gamma function; i.e.

$$
\Phi_{N}(x)=30 x^{2}(1-x)^{2} \quad \text { and } \quad \Phi_{N}(x)=140 x^{3}(1-x)^{3}
$$

for tensor mesons and pseudotensor mesons respectively (notice that, for $L \neq 0$, using the asymptotic DA without modification, i.e. Eq. (22) for $L=0$, would lead to divergences).

Finally, for the pion, we shall use either the asymptotic DA [1] :

$$
\Phi_{N}(x)=6 x(1-x),
$$

or the Chernyak-Zhitnitsky DA [9] 3 :

$$
\Phi_{N}(x)=30 x(1-x)(1-2 x)^{2} .
$$

As for the constants $f_{L}$, we extract them (more precisely : their absolute values) from the known experimental values of the decay widths $\Gamma(Q \rightarrow \gamma \gamma)$, as we compute the corresponding theoretical expressions by applying again the formalism presented in Sect. 2 (see Appendix C).

There is then no free parameter left in the calculation of the amplitudes $\mathcal{M}$ of the various processes considered, except for an irrelevant phase factor and for some limited freedom in the choice of the value of the strong coupling constant.

From those amplitudes the differential cross section with respect to $\cos \Theta$ is derived as follows (neglecting meson masses, as well, in the phase-space factor) :

$$
\frac{d \sigma^{\gamma \gamma^{\prime} \rightarrow Q Q^{\prime}}(E, \Theta)}{d(\cos \Theta)}=\frac{\xi}{128 \pi E^{2}} \sum_{\lambda_{\gamma} \lambda_{\gamma}^{\prime}, \Lambda \Lambda^{\prime}}\left|\mathcal{M}_{\lambda_{\gamma} \lambda_{\gamma}^{\prime}}^{L S J \Lambda L^{\prime} S^{\prime} J^{\prime} \Lambda^{\prime}}(E, \Theta)\right|^{2},
$$

\footnotetext{
${ }^{3}$ We are aware that both of those pion DA's are partly unsatisfactory as regards practical applications : the asymptotic DA does not fit the pion's electromagnetic form factor, while the Chernyak-Zhitnitsky DA is disfavoured because of its incompatibility with the photon-pion transition form factor. We are using them nevertheless, as we did in previous papers [6, [7, since presently no "gold-plated" pion DA is available.
} 
where the coefficient $\xi$ takes the value $1 / 2$ when $Q, Q^{\prime}$ are identical particles, and 1 otherwise.

Details of our calculations, for the three processes $\gamma \gamma \rightarrow Q Q^{\prime}$ considered, are shown in Appendix B. Only for the choice of the nonrelativistic DA are those calculations performed analytically till the end.

Choosing the value $\alpha_{s}=0.3$, we are plotting in Figs. 2 and 3, for the three types of reactions considered and for the known charged or neutral tensor and pseudotensor mesons to be produced in these reactions, the scaling differential cross section $E^{8} d \sigma / d|t|$, which is easily derived from Eq. (26) [noticing that, neglecting masses, $|t|=\left(E^{2} / 2\right)(1-\cos \Theta)$ ]. Figs. 2 (a),(b) correspond to the case of tensor-meson pairs, making use of the nonrelativistic DA [Eq. (21)] and of the generalized asymptotic DA [Eq. (23)] respectively. Similarly, Figs. $3(\mathrm{a})$,(b) show the case of pseudotensor-meson pairs and of hybrid (pion plus pseudotensor meson) pairs.

On the other hand we are interested in the $p_{T}$ distribution of the outgoing mesons. Replacing the variable $\cos \Theta$ by $p_{T}$, we get :

$$
\frac{d \sigma^{\gamma \gamma^{\prime} \rightarrow Q Q^{\prime}}\left(E, p_{T}\right)}{d p_{T}}=\frac{p_{T} \xi}{64 \pi E^{3} \sqrt{p^{2}-p_{T}^{2}}} \sum_{\lambda_{\gamma} \lambda_{\gamma}^{\prime} \Lambda \Lambda^{\prime}}\left[\left|\mathcal{M}_{\lambda_{\gamma} \lambda_{\gamma}^{\prime}}^{\Lambda \Lambda^{\prime}}(E, \Theta)\right|^{2}+\left|\mathcal{M}_{\lambda_{\gamma} \lambda_{\gamma}^{\prime}}^{\Lambda \Lambda^{\prime}}(E, \pi-\Theta)\right|^{2}\right]
$$

where, in the expressions of the amplitudes, $\cos \Theta$ is to be replaced by $\left[1-p_{T}^{2} / p^{2}\right]^{1 / 2}, p^{2}$ being given by

$$
p^{2}=\frac{1}{4 E^{2}}\left[E^{4}-2\left(M^{2}+M^{\prime 2}\right) E^{2}+\left(M^{2}-M^{\prime 2}\right)^{2}\right]
$$

(It is indeed preferable to keep the meson masses finite in the expression of $p^{2}$, if one wishes to extrapolate the $p_{T}$ spectrum to relatively small values of $p_{T}$ ).

From Eq. (27) one derives the transverse-momentum spectrum for the overall reaction $e e^{\prime} \rightarrow e e^{\prime} Q Q^{\prime}:$

$$
\frac{d \sigma^{e e^{\prime} \rightarrow e e^{\prime} Q Q^{\prime}}\left(s, p_{T}\right)}{d p_{T}}=\int_{z_{\text {min }}}^{1} f(z) d z \int_{z_{\text {min }}^{\prime}}^{1} f\left(z^{\prime}\right) d z^{\prime} \frac{d \sigma^{\gamma \gamma^{\prime} \rightarrow Q Q^{\prime}}\left(E, p_{T}\right)}{d p_{T}}
$$

where $s$ is the overall c.m. energy squared ; $f(z)$ resp. $f\left(z^{\prime}\right)$, is the equivalent-photon spectrum of either electron, given by

$$
f(z)=\frac{\alpha}{\pi z}\left[\left(1-z+\frac{z^{2}}{2}\right) \ln \frac{s}{4 \mathrm{~m}_{\mathrm{e}}^{2}}-(1-z)\right]
$$

and we notice that

$$
E^{2}=z z^{\prime} s, \quad z_{\min }^{\prime}=\frac{E_{\min }^{2}}{z s}, \quad z_{\min }=\frac{E_{m i n}^{2}}{s}
$$


where

$$
E_{\text {min }}=\left(p_{T}^{2}+M^{2}\right)^{1 / 2}+\left(p_{T}^{2}+M^{\prime 2}\right)^{1 / 2} .
$$

As for the running strong-interaction coupling constant, we here use the lowest-order (one-loop) formula :

$$
\alpha_{s}=\frac{12 \pi}{25 \ln \left(" Q^{2 "} / \Lambda_{\mathrm{QCD}}^{2}\right)},
$$

where an empirical value of $200 \mathrm{MeV}$ is taken for $\Lambda_{Q C D}$. Regarding the scale " $Q$ ", we associate it, in the spirit of Refs. [10, 11], with the mean absolute value (i.e., the absolute value for $x=x^{\prime}=1 / 2$ ) of the squared four-momentum of the timelike or spacelike gluon exchanged in the Feynman diagrams for $\gamma \gamma \rightarrow q \bar{q} q^{\prime} \bar{q}^{\prime}$; thus " $Q{ }^{2} " \approx E^{2} / 4$.

The numerical integration over $z, z^{\prime}$ and $p_{T}$ leads to the values shown in tables 1-3 for the integrated cross sections, respectively for the three cases : i) $\sqrt{s}=200 \mathrm{GeV}$ (LEP2 energy), $p_{T}>1 \mathrm{GeV}$; ii) $\sqrt{s}=200 \mathrm{GeV}, p_{T}>2 \mathrm{GeV}$; iii) $\sqrt{s}=10 \mathrm{GeV}$ (energy of a "B factory"), $p_{T}>1 \mathrm{GeV}$.

The three types of reactions, and both types of distribution amplitudes here defined for tensor and pseudotensor mesons, are considered.

\section{Conclusion}

As is shown by tables 1-3 (and as appears already in Figs. 2,3), the results obtained do not differ widely depending on the distribution amplitude chosen for tensor resp. pseudotensor mesons. Except for the case of $\pi_{2}^{0} \pi_{2}^{0}$ production, the generalized asymptotic DA leads to approximately equal or slightly (at most by a factor of about 3 ) higher values, as compared with the nonrelativistic one.

On the other hand, comparing the Chernyak-Zhitnitsky distribution amplitude with the asymptotic one for the pion in the case of hybrid-pair production, one notices that the former gives rise to somewhat higher yields (by a factor of 2-4) than the latter.

Finally it should be remarked that in general the charged channels give rise to significantly higher yields than the neutral ones. There appears to be some hope that the production of charged-meson pairs as here considered may become measurable with highenergy $e^{+} e^{-}$colliders of the next generation, provided integrated luminosities as high as $\approx 10^{40} \mathrm{~cm}^{-2}$ can be reached.

\section{Appendix A : Expression of four-vectors and of spinor- pair projectors}

For the four-momenta of initial and final particles, resp. partons, involved in the process $a b \rightarrow Q c$, we get in the c.m. frame of that process [Fig. 1(A)], assuming the masses of 
$a, b, c$ to be zero or negligible, the following expressions (components $0, x, y, z$ in that order) :

$$
\begin{aligned}
& a^{\mu}=\frac{E}{2}\left(\begin{array}{c}
1 \\
-\sin \Theta \\
0 \\
\cos \Theta
\end{array}\right) \quad b^{\mu}=\frac{E}{2}\left(\begin{array}{c}
1 \\
\sin \Theta \\
0 \\
-\cos \Theta
\end{array}\right) \\
& Q^{\mu}=\frac{E}{2}\left(\begin{array}{c}
1+\eta^{2} \\
0 \\
0 \\
1-\eta^{2}
\end{array}\right) \quad c^{\mu}=\frac{E}{2}\left(1-\eta^{2}\right)\left(\begin{array}{c}
1 \\
0 \\
0 \\
-1
\end{array}\right) \text {, }
\end{aligned}
$$

where we have called all four-momenta like the corresponding particles and set $\eta=M / E$.

For the four-momenta of the quarks $q, \bar{q}$ we get, after a Lorentz transformation from the meson rest frame [Fig. 1(B)] to the c.m. frame of the process considered [Fig. 1(A)] :

$$
q^{\mu}=\frac{E x}{2}\left(\begin{array}{c}
1+\beta_{q} \cos \theta+\eta^{2}\left(1-\beta_{q} \cos \theta\right) \\
2 \eta \beta_{q} \sin \theta \cos \phi \\
2 \eta \beta_{q} \sin \theta \sin \phi \\
1+\beta_{q} \cos \theta-\eta^{2}\left(1-\beta_{q} \cos \theta\right)
\end{array}\right) \quad \bar{q}^{\mu}=q^{\mu}\left(x \rightarrow 1-x, \beta_{q} \rightarrow-\beta_{\bar{q}}\right)
$$

where we have set :

$$
\beta_{q}=\frac{k}{M x}=\frac{\beta}{2 x}, \quad \beta_{\bar{q}}=\frac{k}{M(1-x)}=\frac{\beta}{2(1-x)} .
$$

Letting $\eta$ go to zero, Eq. (A1) remains unmodified, while Eqs. (A2], (A3) are replaced by the simplified formulas (A5), (A6) as follows

$$
\begin{aligned}
& Q^{\mu}=\frac{E}{2}\left(\begin{array}{l}
1 \\
0 \\
0 \\
1
\end{array}\right) c^{\mu}=\frac{E}{2}\left(\begin{array}{c}
1 \\
0 \\
0 \\
-1
\end{array}\right) \\
& q^{\mu}=\frac{E x}{2}\left(1+\beta_{q} \cos \theta\right)\left(\begin{array}{l}
1 \\
0 \\
0 \\
1
\end{array}\right) \quad \bar{q}^{\mu}=\frac{E(1-x)}{2}\left(1-\beta_{\bar{q}} \cos \theta\right)\left(\begin{array}{l}
1 \\
0 \\
0 \\
1
\end{array}\right) .
\end{aligned}
$$

From Eq. (A6) one derives the expressions of the projectors of spinor pairs to be used in the computation of the helicity amplitudes at the parton level. We notice that, in the expressions of the quark four-vectors (and consequently as well of the quark spinors), any dependence on $\phi$ has vanished. Therefore, as stated in Sect. 2, all helicity amplitudes $\mathcal{T}$ become independent of $\phi$. Calling $u^{\lambda_{q}}, v^{\lambda_{\bar{q}}}$ the spinors of the quark and antiquark, with helicity $\lambda_{q}$ and $\lambda_{\bar{q}}$ respectively, and introducing the Clebsch-Gordan coefficient $\left\langle\frac{1}{2} \frac{1}{2} \lambda_{q} \lambda_{\bar{q}} \mid \frac{1}{2} \frac{1}{2} S \Lambda\right\rangle$, one gets the four projectors $P_{\Lambda}^{S}$ needed : 


$$
\begin{aligned}
P_{1}^{1} & =v^{1 / 2} \bar{u}^{1 / 2} \\
& =\frac{1}{\sqrt{2}} \sqrt{x(1-x)} \sqrt{\left(1+\beta_{q} \cos \theta\right)\left(1-\beta_{\bar{q}} \cos \theta\right)} \notin_{+}^{*} \varnothing, \\
P_{0}^{1} & =\frac{1}{\sqrt{2}}\left(v^{-1 / 2} \bar{u}^{1 / 2}+v^{1 / 2} \bar{u}^{-1 / 2}\right) \\
& =\frac{1}{\sqrt{2}} \sqrt{x(1-x)} \sqrt{\left(1+\beta_{q} \cos \theta\right)\left(1-\beta_{\bar{q}} \cos \theta\right)} \varnothing, \\
P_{-1}^{1} & =\frac{1}{\sqrt{2}} v^{-1 / 2} \bar{u}^{-1 / 2} \\
& =\frac{1}{\sqrt{2}} \sqrt{x(1-x)} \sqrt{\left(1+\beta_{q} \cos \theta\right)\left(1-\beta_{\bar{q}} \cos \theta\right)} \notin_{-}^{*} \varnothing, \\
P_{0}^{0} & =\frac{1}{\sqrt{2}}\left(v^{-1 / 2} \bar{u}^{1 / 2}-v^{1 / 2} \bar{u}^{-1 / 2}\right) \\
& =\frac{1}{\sqrt{2}} \sqrt{x(1-x)} \sqrt{\left(1+\beta_{q} \cos \theta\right)\left(1-\beta_{\bar{q}} \cos \theta\right)} \gamma_{5} \varnothing .
\end{aligned}
$$

Here the four-vectors $\epsilon_{+}^{*}, \epsilon_{-}^{*}$ are defined in the usual way :

$$
\epsilon_{+}^{* \mu}=-\frac{1}{\sqrt{2}}\left(\begin{array}{c}
0 \\
1 \\
-i \\
0
\end{array}\right) \quad \epsilon_{-}^{* \mu}=\frac{1}{\sqrt{2}}\left(\begin{array}{c}
0 \\
1 \\
i \\
0
\end{array}\right)
$$

When one goes over from the process $a b \rightarrow Q c$ to $a b \rightarrow Q Q^{\prime}, c^{\mu}$ is replaced by $Q^{\prime \mu}$ in Eq. (A5), and in addition the following expressions are to be used for the four-vectors $q^{\prime \mu}, \bar{q}^{\prime \mu}:$

$$
q^{\prime \mu}=\frac{E x^{\prime}}{2}\left(1-\beta_{q^{\prime}}^{\prime} \cos \theta^{\prime}\right)\left(\begin{array}{c}
1 \\
0 \\
0 \\
-1
\end{array}\right) \quad \bar{q}^{\prime \mu}=\frac{E\left(1-x^{\prime}\right)}{\sqrt{2}}\left(1+\beta_{\bar{q}^{\prime}}^{\prime} \cos \theta^{\prime}\right)\left(\begin{array}{c}
1 \\
0 \\
0 \\
-1
\end{array}\right)
$$

defining

$$
\beta_{q^{\prime}}^{\prime}=\frac{\beta^{\prime}}{2 x^{\prime}}, \quad \beta_{\bar{q}^{\prime}}^{\prime}=\frac{\beta^{\prime}}{2\left(1-x^{\prime}\right)} .
$$


As for the corresponding projectors of spinor pairs, $P_{\Lambda^{\prime}}^{\prime S^{\prime}}$, they are written as follows :

$$
\begin{aligned}
P_{1}^{\prime 1} & =\frac{1}{\sqrt{2}} \sqrt{x^{\prime}\left(1-x^{\prime}\right)} \sqrt{\left(1-\beta_{q^{\prime}}^{\prime} \cos \theta^{\prime}\right)\left(1+\beta_{\bar{q}^{\prime}}^{\prime} \cos \theta^{\prime}\right)} \notin_{+}^{*} \varnothing^{\prime}, \\
P_{0}^{\prime 1} & =\frac{1}{\sqrt{2}} \sqrt{x^{\prime}\left(1-x^{\prime}\right)} \sqrt{\left(1-\beta_{q^{\prime}}^{\prime} \cos \theta^{\prime}\right)\left(1+\beta_{\bar{q}^{\prime}}^{\prime} \cos \theta\right)^{\prime}} \mathscr{Q}^{\prime}, \\
P_{-1}^{\prime 1} & =\frac{1}{\sqrt{2}} \sqrt{x^{\prime}\left(1-x^{\prime}\right)} \sqrt{\left(1-\beta_{q}^{\prime} \cos \theta^{\prime}\right)\left(1+\beta_{\bar{q}^{\prime}}^{\prime} \cos \theta^{\prime}\right)} \notin_{-}^{*} \mathscr{Q}^{\prime}, \\
P_{0}^{\prime 0} & =\frac{1}{\sqrt{2}} \sqrt{x^{\prime}\left(1-x^{\prime}\right)} \sqrt{\left(1-\beta_{q^{\prime}}^{\prime} \cos \theta^{\prime}\right)\left(1+\beta_{\bar{q}^{\prime}}^{\prime} \cos \theta\right)^{\prime}} \gamma_{5} \varnothing^{\prime},
\end{aligned}
$$

where one defines : $\epsilon_{ \pm}^{\prime * \mu}=\epsilon_{\mp}^{* \mu}$.

\section{Appendix B : Details of calculation of the processes considered}

Using the ingredients given in Appendix A, we shall here provide details of calculation of the three processes considered in Sect. 3 ; that calculation is based on computing the usual Feynman diagrams involved in the Brodsky-Lepage mechanism [1] for $\gamma \gamma$ production of $(q \bar{q})$ meson pairs (e.g. $\gamma \gamma \rightarrow \pi \pi)$. We shall give hereafter

- except for the case of hybrid-pair production (where they become too lengthy), the general expression of the physically relevant pieces of the helicity amplitudes $\mathcal{T}_{\lambda_{\gamma} \lambda_{\gamma}^{\prime}}^{\Lambda \Lambda \prime^{\prime}}$ (dropping the superscripts $S, S^{\prime}$ of those amplitudes) ;

- the expressions of the amplitudes $\mathcal{M}_{\lambda_{\gamma} \lambda_{\gamma}^{\prime}}^{\Lambda \Lambda^{\prime}}$ (dropping the superscripts $S, L, J, S^{\prime}, L^{\prime}, J^{\prime}$ ), but only for the choice of the nonrelativistic distribution amplitude given by Eq. (21) for $(q \bar{q})$ tensor and pseudotensor mesons ; indeed, using the DA given by Eq. (23), it becomes difficult, if not impossible, to perform the integration over both $x$ and $x^{\prime}$, involved in Eqs. (18)-(20), analytically ;

- the expressions of the differential cross sections $d \sigma^{\gamma \gamma^{\prime} \rightarrow Q Q^{\prime}} / d(\cos \Theta)$, again only for the choice of the nonrelativistic distribution amplitude for tensor and pseudotensor mesons.

\section{(i) $\gamma \gamma$ production of $(\boldsymbol{q} \overline{\boldsymbol{q}})$ tensor-meson pairs}

Keeping only the physically relevant pieces (i.e. the terms in $\beta^{1} \beta^{11}$ of the series expansion in powers of $\left.\beta, \beta^{\prime}\right)$ of the helicity amplitudes $\mathcal{T}_{\lambda_{\gamma} \lambda_{\gamma}^{\prime}}^{\Lambda \Lambda^{\prime}}$, we obtain :

$$
\left(\mathcal{T}_{ \pm \mp}^{+-}\right)_{\text {phys. }}=-\frac{K}{4\left(a^{2}-b^{2}\right)^{2}}\left\{\left\langle\left(e_{1}-e_{2}\right)^{2}\right\rangle(2 a-1)\right.
$$




$$
\begin{aligned}
& \pm 2 v(1 \mp v) \frac{\left\langle e_{1} e_{2}\right\rangle}{\left[a^{2}-v^{2} b^{2}\right]^{3}}\left[a^{2}\left(2 a^{4}-9 a^{2} b^{2}+6 a^{3} b^{2}+6 b^{4}-4 a b^{4}\right)\right. \\
& \left.\left.+2 v^{2} b^{2}\left(3 a^{4}-3 a^{2} b^{2}-4 a^{3} b^{2}+b^{4}+2 a b^{4}\right)+v^{4}(2 a-1) b^{6}\right]\right\} u u^{\prime} \beta \beta^{\prime} \\
& \left(\mathcal{T}_{\mp \pm}^{-+}\right)_{\text {phys. }}=\left(\mathcal{T}_{ \pm \mp}^{+-}\right)_{p h y s .} \\
& \left(\mathcal{T}_{ \pm \pm}^{00}\right)_{\text {phys. }}=-\frac{K}{2\left(a^{2}-b^{2}\right)^{2}} \frac{\left\langle\left(e_{1}-e_{2}\right)^{2}\right\rangle}{1-v^{2}} a u u^{\prime} \beta \beta^{\prime} \\
& \left(\mathcal{T}_{ \pm \mp}^{00}\right)_{\text {phys. }}=\frac{K}{2\left(a^{2}-b^{2}\right)^{2}}\left\{\frac{\left\langle\left(e_{1}-e_{2}\right)^{2}\right\rangle}{1-v^{2}}(1-a)\right. \\
& +\frac{\left\langle e_{1} e_{2}\right\rangle}{\left[a^{2}-v^{2} b^{2}\right]^{3}}\left[a^{3}\left(a^{3}-3 a^{2} b^{2}+2 b^{4}\right)\right. \\
& +2 v^{2} a\left(-a^{5}+3 a^{3} b^{2}+3 a^{4} b^{2}-3 a b^{4}-5 a^{2} b^{4}+3 b^{6}\right) \\
& \left.\left.+v^{4} b^{2}\left(-6 a^{4}+9 a^{2} b^{2}+2 a^{3} b^{2}-2 b^{4}-3 a b^{4}\right)\right]\right\} u u^{\prime} \beta \beta^{\prime}
\end{aligned}
$$

all other relevant helicity amplitudes $\mathcal{T}_{\lambda_{\gamma} \lambda_{\gamma}^{\prime}}^{\Lambda \Lambda^{\prime}}$ being zero. In the above equations we have introduced the constant $K=2^{10} \pi^{2} \alpha \alpha_{s} /\left(3 E^{2}\right)$, using also for shorthand $v=\cos \Theta, u=$ $\cos \theta, u^{\prime}=\cos \theta^{\prime}$. In addition we have put

$$
\begin{aligned}
& a=(1-x)\left(1-x^{\prime}\right)+x x^{\prime} \\
& b=(1-x)\left(1-x^{\prime}\right)-x x^{\prime}
\end{aligned}
$$

In standard notation, $e_{1}$ and $-e_{2}$ are the charges, in units of the proton charge, of the quark and the antiquark of which a given meson is composed ; $\left\langle e_{1} e_{2}\right\rangle$ and $\left\langle\left(e_{1}-e_{2}\right)^{2}\right\rangle$ are then charge factors, adequately convoluted with the flavor components of the $Q, Q^{\prime}$ meson wave functions. The numerical values of these charge factors for the various meson pairs here considered are given in table B1.

Therefrom we get through Eq. (18), with the choice $\Phi_{N}(x)=\delta(x-1 / 2), \Phi_{N}^{\prime}\left(x^{\prime}\right)=$ $\delta\left(x^{\prime}-1 / 2\right):$

$$
\begin{aligned}
& \mathcal{M}_{ \pm \mp}^{+-}=\mp \frac{8}{9} K f_{1} f_{1}^{\prime}\left\langle e_{1} e_{2}\right\rangle v(1 \mp v) \\
& \mathcal{M}_{\mp \pm}^{-+}=\mathcal{M}_{ \pm \mp}^{+-} \\
& \mathcal{M}_{ \pm \pm}^{00}=-\frac{8}{27} K f_{1} f_{1}^{\prime} \frac{\left\langle\left(e_{1}-e_{2}\right)^{2}\right\rangle}{1-v^{2}}
\end{aligned}
$$




$$
\mathcal{M}_{ \pm \mp}^{00}=\frac{8}{27} K f_{1} f_{1}^{\prime}\left[\frac{\left\langle\left(e_{1}-e_{2}\right)^{2}\right\rangle}{1-v^{2}}+2\left\langle e_{1} e_{2}\right\rangle\left(1-2 v^{2}\right)\right] .
$$

Finally, with the choice of the nonrelativistic DA for the tensor mesons, Eq. (26) leads us to :

$$
\begin{aligned}
\frac{d \sigma^{\gamma \gamma \rightarrow Q Q^{\prime}}}{d(\cos \Theta)} & =\frac{2^{21} \pi^{3} \alpha^{2} \alpha_{s}^{2}\left|f_{1}\right|^{2}\left|f_{1}^{\prime}\right|^{2} \xi}{3^{8} s^{3}}\left\{\frac{\left\langle\left(e_{1}-e_{2}\right)^{2}\right\rangle^{2}}{\left(1-v^{2}\right)^{2}}\right. \\
& \left.+2\left\langle e_{1} e_{2}\right\rangle\left\langle\left(e_{1}-e_{2}\right)^{2}\right\rangle \frac{1-2 v^{2}}{1-v^{2}}+\left\langle e_{1} e_{2}\right\rangle^{2}\left(2+v^{2}+17 v^{4}\right)\right\} .
\end{aligned}
$$

\section{(ii) $\gamma \gamma$ production of $(\boldsymbol{q} \overline{\boldsymbol{q}})$ pseudotensor-meson pairs}

Keeping only the physically relevant pieces (i.e. the terms in $\beta^{2} \beta^{2}$ of the series expansion in powers of $\beta, \beta^{\prime}$ ) of the helicity amplitudes $\mathcal{T}_{\lambda_{\gamma} \lambda_{\gamma}^{\prime}}^{\Lambda \Lambda^{\prime}}$, we obtain :

$$
\begin{aligned}
\left(\mathcal{T}_{ \pm \pm}^{00}\right)_{\text {phys. }}=\frac{K}{32} \frac{\left\langle\left(e_{1}-e_{2}\right)^{2}\right\rangle}{1-v^{2}} \frac{8 a^{2}-3 a+4 b^{2}}{\left(a^{2}-b^{2}\right)^{3}} u^{2} u^{\prime 2} \beta^{2} \beta^{2} \\
\left(\mathcal{T}_{ \pm \mp}^{00}\right)_{p h y s .}=\frac{K}{32} \frac{1}{\left(a^{2}-b^{2}\right)^{3}}\left\{\frac{\left\langle\left(e_{1}-e_{2}\right)^{2}\right\rangle}{1-v^{2}}\left(8 a^{2}+4 b^{2}-21 a+9\right)-3 \frac{\left\langle e_{1} e_{2}\right\rangle}{\left(a^{2}-v^{2} b^{2}\right)^{5}}\right. \\
\times \quad\left[a ^ { 5 } \left(4 a^{6}-3 a^{5}-16 a^{5} b^{2}+15 a^{4} b^{2}+20 a^{3} b^{4}-20 a^{2} b^{4}-8 a b^{6}\right.\right. \\
\left.+8 b^{6}\right)+4 v^{2} a^{3}\left(-2 a^{8}+3 a^{7}+8 a^{7} b^{2}-5 a^{6} b^{2}-15 a^{5} b^{2}-20 a^{5} b^{4}\right. \\
\left.+25 a^{4} b^{4}+25 a^{3} b^{4}+26 a^{3} b^{6}-45 a^{2} b^{6}-10 a b^{6}-10 a b^{8}+20 b^{8}\right) \\
+2 v^{4} a\left(-4 a^{9}+70 a^{7} b^{2}-70 a^{6} b^{4}-165 a^{5} b^{4}+8 a^{5} b^{6}+181 a^{4} b^{6}\right. \\
\left.+130 a^{3} b^{6}-40 a^{3} b^{8}-110 a^{2} b^{8}-40 a b^{8}+20 a b^{10}+20 b^{10}\right) \\
+4 v^{6} b^{2}\left(-20 a^{8}+30 a^{7} b^{2}+65 a^{6} b^{2}-8 a^{6} b^{4}-79 a^{5} b^{4}-75 a^{4} b^{4}\right. \\
\left.+20 a^{4} b^{6}+65 a^{3} b^{6}+35 a^{2} b^{6}-10 a^{2} b^{8}-23 a b^{8}-2 b^{8}+2 b^{10}\right) \\
+v^{8} b^{4}\left(-40 a^{6}+16 a^{5} b^{2}+100 a^{4} b^{2}-40 a^{3} b^{4}-75 a^{2} b^{4}+31 a b^{6}\right. \\
\left.\left.\left.+12 b^{6}-4 b^{8}\right)\right]\right\} u^{2} u^{\prime 2} \beta^{2} \beta^{\prime 2} .
\end{aligned}
$$

Therefrom we get through Eq. (19), with the choice $\Phi_{N}(x)=\delta(x-1 / 2), \Phi_{N}^{\prime}\left(x^{\prime}\right)=$ $\delta\left(x^{\prime}-1 / 2\right):$

$$
\begin{gathered}
\mathcal{M}_{ \pm \pm}^{00}=\frac{4}{225} K f_{2} f_{2}^{\prime} \frac{\left\langle\left(e_{1}-e_{2}\right)^{2}\right\rangle}{1-v^{2}} \\
\mathcal{M}_{ \pm \mp}^{00}=\frac{4}{225} K f_{2} f_{2}^{\prime}\left\{\frac{\left\langle\left(e_{1}-e_{2}\right)^{2}\right\rangle}{1-v^{2}}+6\left\langle e_{1} e_{2}\right\rangle\left[1-8 v^{2}\left(1-v^{2}\right)\right]\right\}
\end{gathered}
$$

Finally, with the choice of the nonrelativistic DA for the pseudotensor mesons, Eq. (26) leads us to : 


$$
\begin{aligned}
\frac{d \sigma^{\gamma \gamma \rightarrow Q Q^{\prime}}}{d(\cos \Theta)} & =\frac{2^{19} \pi^{3} \alpha^{2} \alpha_{s}^{2}\left|f_{2}\right|^{2}\left|f_{2}^{\prime}\right|^{2} \xi}{3^{6} 5^{4} s^{3}}\left\{\frac{\left\langle\left(e_{1}-e_{2}\right)^{2}\right\rangle^{2}}{\left(1-v^{2}\right)^{2}}+6\left\langle e_{1} e_{2}\right\rangle\left\langle\left(e_{1}-e_{2}\right)^{2}\right\rangle \frac{1-8 v^{2}\left(1-v^{2}\right)}{1-v^{2}}\right. \\
& \left.+18\left\langle e_{1} e_{2}\right\rangle^{2}\left[1-8 v^{2}\left(1-v^{2}\right)\right]^{2}\right\}
\end{aligned}
$$

\section{(iii) $\gamma \gamma$ production of a pion plus a $(q \bar{q})$ pseudotensor meson}

Let us identify $Q$ with the pion, $Q^{\prime}$ with the pseudotensor meson. Since for that process the expressions of the helicity amplitudes $\mathcal{T}_{\lambda_{\gamma} \lambda_{\gamma}^{\prime}}^{\Lambda \Lambda^{\prime}}$ (even keeping only the physically relevant pieces, i.e. the terms in $\beta^{0} \beta^{\prime 2}$ of the series expansion in powers of $\beta, \beta^{\prime}$ ) become particularly lengthy, we shall not show them here. We shall thus restrict ourselves to giving the expressions of the amplitudes $\mathcal{M}_{\lambda_{\gamma} \lambda_{\gamma}^{\prime}}^{\Lambda \Lambda^{\prime}}$ obtained with the choice of the nonrelativistic DA for the pseudotensor meson together with the Chernyak-Zhitnitsky DA for the pion, as well as of the differential cross section derived therefrom through Eq. (26) :

$$
\begin{gathered}
\mathcal{M}_{ \pm \pm}^{00}=\frac{1}{3 \sqrt{3}} K f_{\pi} f_{2}^{\prime} \frac{\left\langle\left(e_{1}-e_{2}\right)^{2}\right\rangle}{1-v^{2}}, \\
\mathcal{M}_{ \pm \mp}^{00}=\frac{1}{3 \sqrt{3}} K f_{\pi} f_{2}^{\prime}\left\{\frac{\left\langle\left(e_{1}-e_{2}\right)^{2}\right\rangle}{1-v^{2}}+3 \frac{\left\langle e_{1} e_{2}\right\rangle}{2 v^{7}}\left[60 v-106 v^{3}+42 v^{5}\right.\right. \\
\left.\left.+\left(30-63 v^{2}+36 v^{4}-4 v^{6}\right) \ln \left(\frac{1-v}{1+v}\right)\right]\right\} \\
\frac{d \sigma^{\gamma \gamma \rightarrow Q Q^{\prime}}}{d(\cos \Theta)}=\frac{2^{14} \pi^{3} \alpha^{2} \alpha_{s}^{2}\left|f_{\pi}\right|^{2}\left|f_{2}^{\prime}\right|^{2} \xi}{3^{5} s^{3}}\left\{2 \frac{\left\langle\left(e_{1}-e_{2}\right)^{2}\right\rangle^{2}}{\left(1-v^{2}\right)^{2}}+6 \frac{\left\langle e_{1} e_{2}\right\rangle\left\langle\left(e_{1}-e_{2}\right)^{2}\right\rangle}{2 v^{7}}\right. \\
\times \quad\left[60 v-106 v^{3}+42 v^{5}+\left(30-63 v^{2}+36 v^{4}-4 v^{6}\right) \ln \left(\frac{1-v}{1+v}\right)\right] \\
\left.+9 \frac{\left\langle e_{1} e_{2}\right\rangle^{2}}{4 v^{14}}\left[60 v-106 v^{3}+42 v^{5}+\left(30-63 v^{2}+36 v^{4}-4 v^{6}\right) \ln \left(\frac{1-v}{1+v}\right)\right]^{2}\right\} .
\end{gathered}
$$

\section{Appendix C : Determination of the normalization con- stants $\left|f_{L}\right|$}

The normalization constants $\left|f_{L}\right|$ for the various mesons $Q$ considered in this paper (except for the pion) are determined through the experimental values of the corresponding decay widths $\Gamma(Q \rightarrow \gamma \gamma)$, using for the theoretical computation the formalism developed in Sec. 2 , but this time staying in the meson rest frame.

Let us define the helicity amplitude $\mathcal{M}_{\lambda_{\gamma} \lambda_{\gamma}^{\prime}}^{L S \Lambda}(\Theta, \Phi)$ for the decay of a meson $Q$ with quantum numbers $L, S, J, \Lambda$ into a pair of photons ; here $\Theta, \Phi$ specify the direction of one 
of the outgoing photons in the meson rest frame. The dependence of $\mathcal{M}_{\lambda_{\gamma} \lambda_{\gamma}^{\prime}}^{L S J \Lambda}(\Theta, \Phi)$ on $\Theta$, $\Phi$ is known a priori [12] :

$$
\mathcal{M}_{\lambda_{\gamma} \lambda_{\gamma}^{\prime}}^{L S S \Lambda}(\Theta, \Phi)=\hat{\mathcal{M}}_{\lambda_{\gamma} \lambda_{\gamma}^{\prime}} d_{\Lambda, \lambda_{\gamma}-\lambda_{\gamma}^{\prime}}^{J}(\Theta) \exp [i \Lambda \Phi]
$$

where the "reduced" amplitude $\hat{\mathcal{M}}_{\lambda_{\gamma} \lambda_{\gamma}^{\prime}}$ is independent of $\Lambda, \Theta$, and $\Phi$. The decay width $\Gamma(Q \rightarrow \gamma \gamma)$ is then given as a function of the reduced amplitudes $\hat{\mathcal{M}}_{\lambda_{\gamma} \lambda_{\gamma}^{\prime}}$ by the relation

$$
\Gamma(Q \rightarrow \gamma \gamma)=\frac{1}{160 \pi M} \sum_{\lambda_{\gamma}, \lambda_{\gamma}^{\prime}}\left|\hat{\mathcal{M}}_{\lambda_{\gamma} \lambda_{\gamma}^{\prime}}\right|^{2} .
$$

The equation to be used, in order to derive the helicity amplitudes at the hadron level, $\mathcal{M}_{\lambda_{\gamma} \lambda_{\gamma}^{\prime}}^{L S J \Lambda}(\Theta, \Phi)$, from the amplitudes $\mathcal{T}_{\lambda_{\gamma} \lambda_{\gamma}^{\prime}}^{S \Lambda_{S}}(\Theta, \Phi, \beta, \theta, \phi, x)$ defined for the corresponding parton process $\left(q \bar{q} \rightarrow \gamma \gamma^{\prime}\right)$, with $\beta, x$ defined as in Sect. 2 and $\theta, \phi$ specifying the direction of the incoming quark in the meson rest frame, is quite similar to Eq. (16) :

$$
\begin{aligned}
\mathcal{M}_{\lambda_{\gamma} \lambda_{\gamma^{\prime}}}^{L S J \Lambda}(\Theta, \Phi) & =f_{L} \lim _{\beta \rightarrow 0} \frac{1}{\beta^{L}} \int \frac{d(\cos \theta)}{2} \int \frac{d \phi}{2 \pi} \sum_{\Lambda_{S}}\left[\zeta^{L S J \Lambda_{L} \Lambda_{S}}(\theta, \phi)\right]^{*} \\
& \times \int \frac{\Phi_{N}(x) d x}{\sqrt{x(1-x)}} \mathcal{T}_{\lambda_{\gamma} \lambda_{\gamma}^{\prime}}^{S \Lambda_{S}}(\Theta, \Phi, \beta, \theta, \phi, x)
\end{aligned}
$$

where $f_{L}$ and $\zeta^{L S J \Lambda_{L} \Lambda_{S}}(\theta, \phi)$ are given, respectively, in Eqs. (15) [combined with Eq. (13)] and (9).

We may choose the meson decay axis as our $z$-axis, thus setting $\Theta=\Phi=0$ and consequently identifying the hadronic helicity amplitudes with the corresponding "reduced" ones [see Eq. (C1)] ; on the other hand, since the reaction at parton level, as seen in three-momentum space, occurs in a plane, we may set $\phi=0$, so that Eq. (C3) simplifies as follows :

$$
\begin{aligned}
\hat{\mathcal{M}}_{\lambda_{\gamma} \lambda_{\gamma^{\prime}}} & =f_{L} \lim _{\beta \rightarrow 0} \frac{1}{\beta^{L}} \int \frac{d(\cos \theta)}{2} \sum_{\Lambda_{S}} \zeta^{\Lambda_{L} \Lambda_{S}}(\theta, 0) \\
& \times \int \frac{\Phi_{N}(x) d x}{\sqrt{x(1-x)}} \mathcal{T}_{\lambda_{\gamma} \lambda_{\gamma}^{\prime}}^{\Lambda_{S}}(\beta, \theta, x),
\end{aligned}
$$

where we have dropped the superscripts $L, S, J$ from $\zeta$ and $S$ from $\mathcal{T}$, and where we notice that $\Lambda_{L}+\Lambda_{S}=\lambda_{\gamma}-\lambda_{\gamma}^{\prime}$, while $\theta$ is (except for an irrelevant change of sign) the angle between incoming quark and outgoing photon in the c.m. frame of the parton process.

In order to evaluate the helicity amplitudes at parton level, $\mathcal{T}_{\lambda_{\gamma} \lambda_{\gamma}^{\prime}}^{\Lambda_{S}}$, we must consider the two Feynman graphs shown in Fig. 4. Before presenting the results in the particular cases of interest, let us comment briefly on two problems connected with the use of a running mass (depending on $x$ ) for the quark and the antiquark. 
i) Gauge invariance is no longer preserved ; however, it can be restored by adding a non-physical term to the photon-quark coupling, i.e. by replacing, at each photonquark vertex, $\gamma^{\mu}$ by $\gamma^{\mu}-\left[\kappa \kappa \kappa^{\mu} / \kappa^{2}\right]_{\kappa^{2} \rightarrow 0}$, where $\kappa$ is the four-momentum of the photon involved. This way, each vertex becomes gauge-invariant by itself.

ii) There is an ambiguity regarding the value of the quark mass in the propagator of either diagram of Fig. 4, since for that value one may choose either $m=M \sqrt{x^{2}-\beta^{2} / 4}$ or $\bar{m}=M \sqrt{(1-x)^{2}-\beta^{2} / 4}$ [see Eq. (3)]. However, as we have checked explicitly, both choices give the same results for the helicity amplitudes at the hadron level (i.e. after integration over $x$ ). This is clearly related to the symmetry of the Feynmangraph calculation and of $\Phi_{N}$ with respect to the exchange $x \longleftrightarrow 1-x$.

Notice that, when the nonrelativistic DA is used, both problems are suppressed anyway. Let us now consider separately the case of tensor and pseudotensor mesons.

\section{(i) Tensor mesons}

In this case the helicity amplitudes at parton level, $\mathcal{T}_{\lambda_{\gamma} \lambda_{\gamma}^{\prime}}^{\Lambda_{S}}$, are given, up to the first order in the $\beta$ power expansion, by the following expressions (amplitudes not given explicitly here are vanishing) :

$$
\begin{gathered}
\mathcal{T}_{++}^{ \pm 1}= \pm \frac{1}{4} K^{\prime} \frac{4 x^{2}-6 x+1}{x \sqrt{x(1-x)}} \beta \sin \theta \\
\mathcal{T}_{--}^{ \pm 1}=\mathcal{T}_{++}^{ \pm 1}, \\
\mathcal{T}_{+-}^{1}=-\mathcal{T}_{-+}^{-1}=2 K^{\prime} \frac{1-x}{\sqrt{x(1-x)}} \beta \sin \theta, \\
\mathcal{T}_{ \pm \pm}^{0}=\frac{1}{2 \sqrt{2}} K^{\prime} \frac{1}{x \sqrt{x(1-x)}} \beta \cos \theta,
\end{gathered}
$$

where $K^{\prime}=8 \sqrt{3} \pi \alpha\left\langle e_{q}^{2}\right\rangle$, with $\left\langle e_{q}^{2}\right\rangle=5 /(9 \sqrt{2}), 1 /(3 \sqrt{2})$, and $1 / 9$ respectively for $f_{2}(1270)$, $a_{2}(1320)$, and $f_{2}^{\prime}(1525)$.

Inserting Eqs. (C5)-(C8) into Eq. (C4), taken for $L=S=1, J=2$, we get the following expressions for the reduced hadronic amplitudes $\hat{\mathcal{M}}_{\lambda_{\gamma} \lambda_{\gamma}^{\prime}}$ :

$$
\begin{gathered}
\hat{\mathcal{M}}_{ \pm \pm}=\frac{1}{3 \sqrt{3}} K^{\prime} f_{1} \int_{0}^{1} d x \Phi_{N}(x) \frac{1-2 x}{x^{2}} \\
\hat{\mathcal{M}}_{ \pm \mp}=-\frac{2 \sqrt{2}}{3} K^{\prime} f_{1} \int_{0}^{1} d x \frac{\Phi_{N}(x)}{1-x} .
\end{gathered}
$$

Finally, using Eq. (C2), and adopting a normalized meson distribution amplitude $\Phi_{N}(x)$ of the kind 


$$
\Phi_{N}^{a}(x)=\frac{2^{2 a+1} \Gamma(a+3 / 2)}{\sqrt{\pi} \Gamma(a+1)} x^{a}(1-x)^{a},
$$

where $a \geq 1+L=2$, we find

$$
\Gamma^{a}(Q \rightarrow \gamma \gamma)=\frac{16}{45} \frac{(2 a+1)^{2}\left(6 a^{2}-12 a+7\right)}{a^{2}(a-1)^{2}} \pi \alpha^{2}\left\langle e_{q}^{2}\right\rangle^{2} \frac{1}{M}\left|f_{1}\right|^{2} .
$$

In particular, for $a=2$, which means [see Eq. (23)] using the generalized asymptotic DA for tensor mesons, Eq. (C12) gives

$$
\Gamma(Q \rightarrow \gamma \gamma)=\frac{140}{9} \pi \alpha^{2}\left\langle e_{q}^{2}\right\rangle^{2} \frac{1}{M}\left|f_{1}\right|^{2}
$$

while for $a \rightarrow \infty$ we recover the nonrelativistic DA [i.e. $\Phi_{N}(x)=\delta(x-1 / 2)$ ], which gives in turn

$$
\Gamma(Q \rightarrow \gamma \gamma)=\frac{128}{15} \pi \alpha^{2}\left\langle e_{q}^{2}\right\rangle^{2} \frac{1}{M}\left|f_{1}\right|^{2}
$$

Making use of the available experimental data for the masses and the two-photon decay widths of the $f_{2}, a_{2}$, and $f_{2}^{\prime}$ tensor mesons [13 and of Eqs. (C13), (C14), one gets estimates of the absolute values of the corresponding $f_{1}$ constants. The results of interest for the calculations of this paper are presented in Tab. C1.

\section{(ii) Pseudotensor mesons}

Here the only non-zero ones among the relevant helicity amplitudes $\mathcal{T}_{\lambda_{\gamma} \lambda_{\gamma}^{\prime}}^{\Lambda_{S}}$ are given, up to order $\beta^{2}$, by the following expression :

$$
\mathcal{T}_{ \pm \pm}^{0}= \pm \sqrt{2} K^{\prime} \frac{1-x}{\sqrt{x(1-x)}} \mp \frac{1}{16 \sqrt{2}} K^{\prime} \frac{1-4(1-x) \cos ^{2} \theta}{x^{2}(1-x) \sqrt{x(1-x)}} \beta^{2}
$$

Inserting Eq. (C15) into Eq. (C4), taken for $L=2, S=0, J=2$, we get the following expression for the only non-vanishing ones among the helicity amplitudes at the hadron level :

$$
\hat{\mathcal{M}}_{ \pm \pm}= \pm \frac{1}{30 \sqrt{2}} K^{\prime} f_{2} \int_{0}^{1} d x \frac{\Phi_{N}(x)}{x^{3}(1-x)} .
$$

Using again the distribution amplitude given in Eq. (C11), but this time with $a \geq$ $1+L=3$, and Eq. (C2) we find

$$
\Gamma^{a}(Q \rightarrow \gamma \gamma)=\frac{8}{375} \frac{\left(4 a^{2}-1\right)^{2}}{a^{2}(a-2)^{2}} \pi \alpha^{2}\left\langle e_{q}^{2}\right\rangle^{2} \frac{1}{M}\left|f_{2}\right|^{2} .
$$

In particular, for $a=3$, which means [see Eq. (23)] choosing the generalized asymptotic DA for pseudotensor mesons, Eq. (C17) gives

$$
\Gamma(Q \rightarrow \gamma \gamma)=\frac{392}{135} \pi \alpha^{2}\left\langle e_{q}^{2}\right\rangle^{2} \frac{1}{M}\left|f_{2}\right|^{2},
$$


while for $a \rightarrow \infty$, which corresponds to the nonrelativistic DA, we find

$$
\Gamma(Q \rightarrow \gamma \gamma)=\frac{128}{375} \pi \alpha^{2}\left\langle e_{q}^{2}\right\rangle^{2} \frac{1}{M}\left|f_{2}\right|^{2},
$$

where, for $\pi_{2}(1670),\left\langle e_{q}^{2}\right\rangle=1 /(3 \sqrt{2})$.

From these relations and from the available experimental data [13] t we can estimate the absolute value of the $f_{2}$ constant for the $\pi_{2}$ pseudotensor meson. The results are presented in Tab. C1.

\section{Acknowledgements}

Two of us (J.H. and F.M.) wish to thank the Laboratoire de Physique Corpusculaire of the Collège de France for the warm hospitality extended to them during a visit in summer 1996, when part of this work was realized. This work has been partially supported by the EU program "Human Capital and Mobility" under contract CHRX-CT94-0450.

\footnotetext{
${ }^{4}$ It should be noticed that the value there given for $\Gamma\left(\pi_{2} \rightarrow \gamma \gamma\right)$, namely $1.35 \mathrm{keV}$, which is based on experimental data from the CELLO [14] and Crystal Ball 15 Collaborations at DESY, has very recently been contested by the ARGUS Collaboration [16] ; this makes our numerical results for pseudotensor-meson pair production, as well as for hybrid pair production, somewhat more controversial.
} 


\section{References}

[1] G.P. Lepage, S.J. Brodsky : Phys. Rev. D22, 2157 (1980) ; S.J. Brodsky, G.P. Lepage : ibid. 24, 1808 (1981) ; 24, 2948 (1981).

[2] See, e.g., S.J. Brodsky, in Proc. of the IX International Workshop on Photon-Photon Collisions, San Diego 1992, eds. D.O. Caldwell and H.P. Paar (World Scientific, Singapore, 1992), p. 209.

[3] R.N. Cahn : Phys. Rev. D35, 3342 (1987).

[4] E.H. Kada, P. Kessler, J. Parisi : Phys. Rev. D39, 2657 (1989).

[5] L. Houra-Yaou, P. Kessler, J. Parisi : Phys. Rev. D45, 794 (1992).

[6] A. Ichola, J. Parisi : Z. Phys. C66, 653 (1995).

[7] F. Murgia, P. Kessler, J. Parisi : Z. Phys. C71, 483 (1996).

[8] M. Benayoun, M. Froissart : Nucl. Phys. B315, 295 (1989).

[9] V.L. Chernyak, A.R. Zhitnitsky : Nucl. Phys. B201, 492 (1982).

[10] S.J. Brodsky, G.P. Lepage, P.B. Mackenzie : Phys. Rev. D28, 228 (1983).

[11] C.R. Ji, F. Amiri : Phys. Rev. D42, 3764 (1990).

[12] See, e.g., C. Bourrely, E. Leader, J. Soffer : Phys. Rep. 59, 95 (1980).

[13] Particle Data Group, R.M. Barnett et al. : Phys. Rev. D54, 1 (1996).

[14] CELLO Collaboration, H.J. Behrend et al. : Z. Phys. C46, 583 (1990).

[15] Crystal Ball Collaboration, D. Antreasyan et al. : Z. Phys. C48, 561 (1990).

[16] ARGUS Collaboration, H. Albrecht et al. : Report DESY 96-112, June 1996. 


\section{Figure captions}

Fig. 1 Kinematic schemes for $(\mathrm{A})$ the process $a b \rightarrow Q c$ in its center-of-mass frame ; $(\mathrm{B})$ the process $a b \rightarrow q \bar{q} c$ in the c.m. frame of $q$ and $\bar{q}$ (meson rest frame).

Fig. 2 Differential cross section $E^{8}[d \sigma / d t]$ in $\mathrm{nb} \times \mathrm{GeV}^{6}$, as a function of $\cos ^{2} \Theta$, for the process $\gamma \gamma \rightarrow Q Q^{\prime}$ involving the production of tensor-meson pairs. The nonrelativistic DA [Eq. (21)] was used for the tensor mesons in Fig. 2(a), while the generalized asymptotic DA [Eq. (23)] was applied for them in Fig. 2(b). For comparison, analogous curves for $\gamma \gamma \rightarrow \pi \pi$, derived in the Brodsky-Lepage formalism, are also shown, using for the pion either the Chernyak-Zhitnitsky DA [Eq. (25)] or the asymptotic DA [Eq. (24)] (Figs. 2(a), 2(b) respectively).

Fig. 3 Differential cross section $E^{8}[d \sigma / d t]$ in $n b \times \mathrm{GeV}^{6}$, as a function of $\cos ^{2} \Theta$, for the process $\gamma \gamma \rightarrow Q Q^{\prime}$ involving the production of pseudotensor-meson and hybrid (one pion plus one pseudotensor meson) pairs. Both the asymptotic DA [Eq. (24), dashed curves] and the Chernyak-Zhitnitsky DA [Eq. (25), full curves] were used for the pion, while for the pseudotensor meson the nonrelativistic DA [Eq. (21)] was applied in Fig. 3(a) and the generalized asymptotic DA [Eq. (23)] in Fig. 3(b).

Fig. 4 Lowest-order Feynman graphs for the process $q \bar{q} \rightarrow \gamma \gamma$. 


\section{Table captions}

Tab. 1 Integrated cross sections (in $10^{-40} \mathrm{~cm}^{2}$ ) of the process $e e^{\prime} \rightarrow e e^{\prime} Q Q^{\prime}$, involving the production of tensor-meson, pseudotensor-meson and hybrid (one pion plus one pseudotensor meson) pairs. For tensor and pseudotensor mesons, both the nonrelativistic DA [Eq. (21)] and the generalized asymptotic DA [Eq. (23)] were used (columns NR and GASY respectively); on the other hand both the asymptotic DA [Eq. (24)] and the Chernyak-Zhitnitsky DA [Eq. (25)] were considered for the pion. Experimental conditions here assumed are $: \sqrt{s}=200 \mathrm{GeV}, p_{T}>1 \mathrm{GeV}$.

Tab. 2 Same as table 1 , but assuming $p_{T}>2 \mathrm{GeV}$.

Tab. 3 Same as table 1, but assuming : $\sqrt{s}=10 \mathrm{GeV}, p_{T}>1 \mathrm{GeV}$.

Tab. B1 Values of the charge factors $\left\langle\left(e_{1}-e_{2}\right)^{2}\right\rangle$ and $\left\langle e_{1} e_{2}\right\rangle$ for the meson pairs considered in this paper.

Tab. C1 Experimental two-photon decay widths, according to Ref. [13], of the known $(q \bar{q})$ tensor and pseudotensor mesons, and values of the normalization constant $\left|f_{L}\right|$ derived therefrom, using either the nonrelativistic DA [Eq. (21)] or the generalized asymptotic DA [Eq. (23)] (columns NR and GASY respectively). 

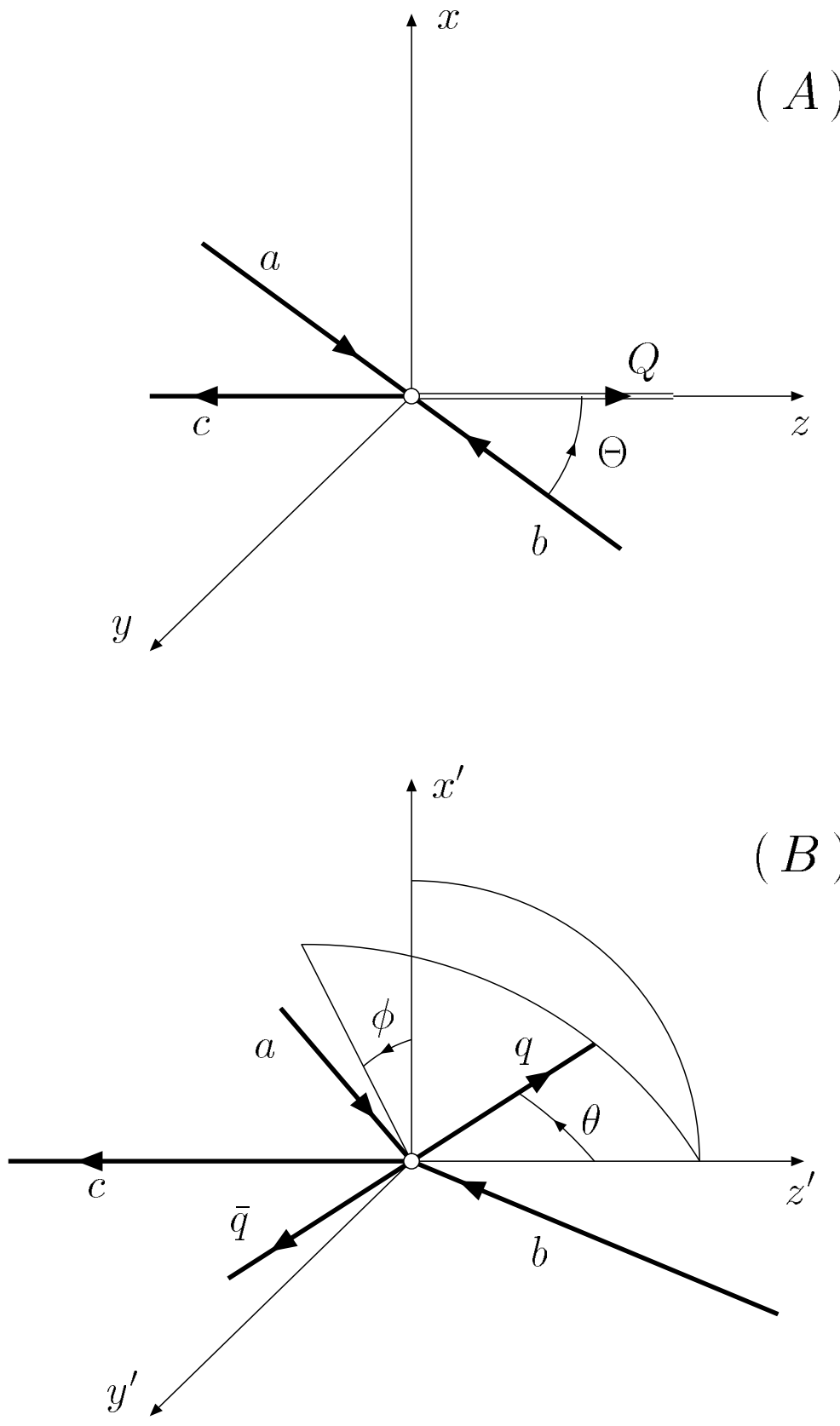

FIG. 1 


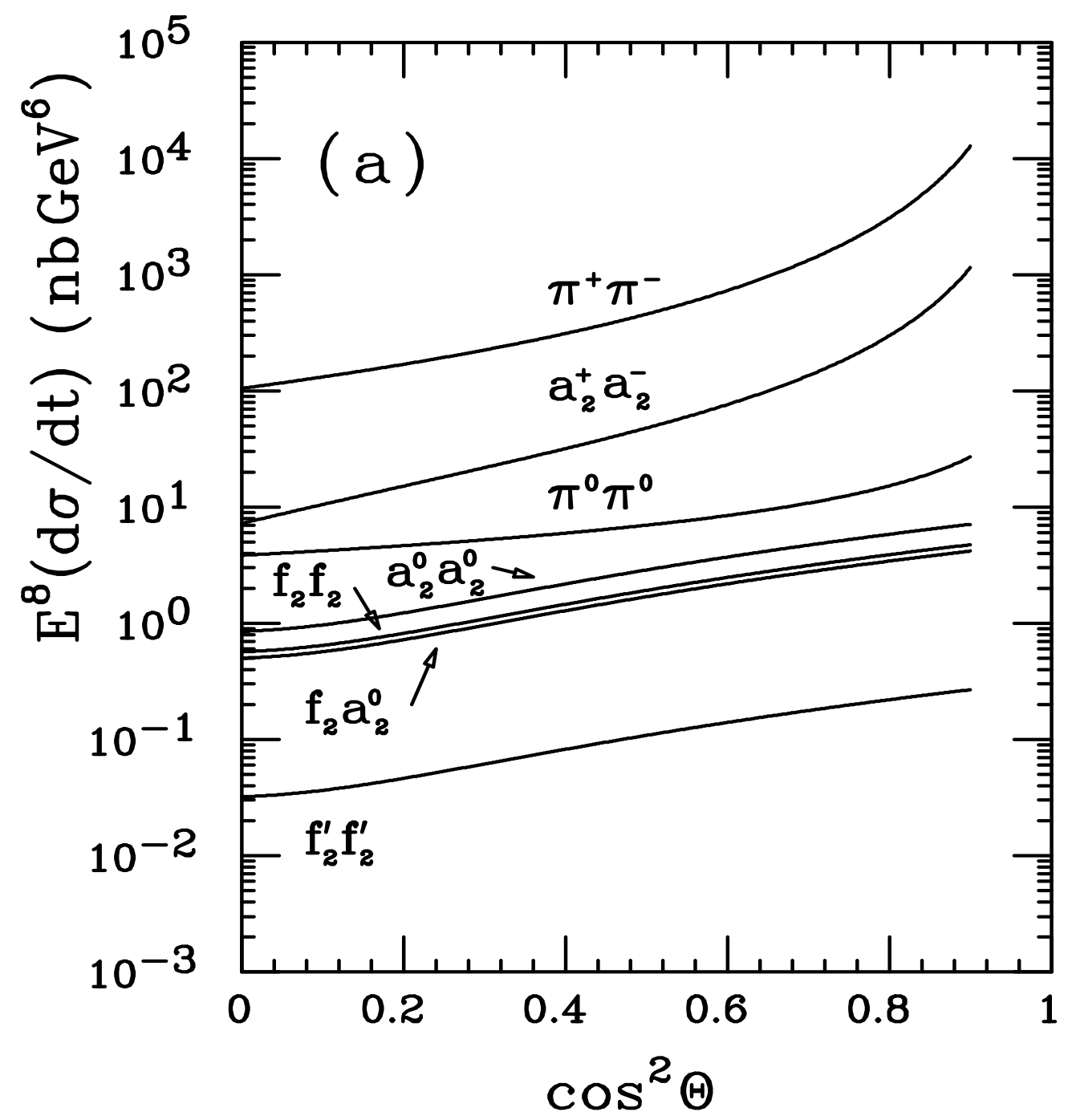

FIG. $2(\mathrm{a})$ 


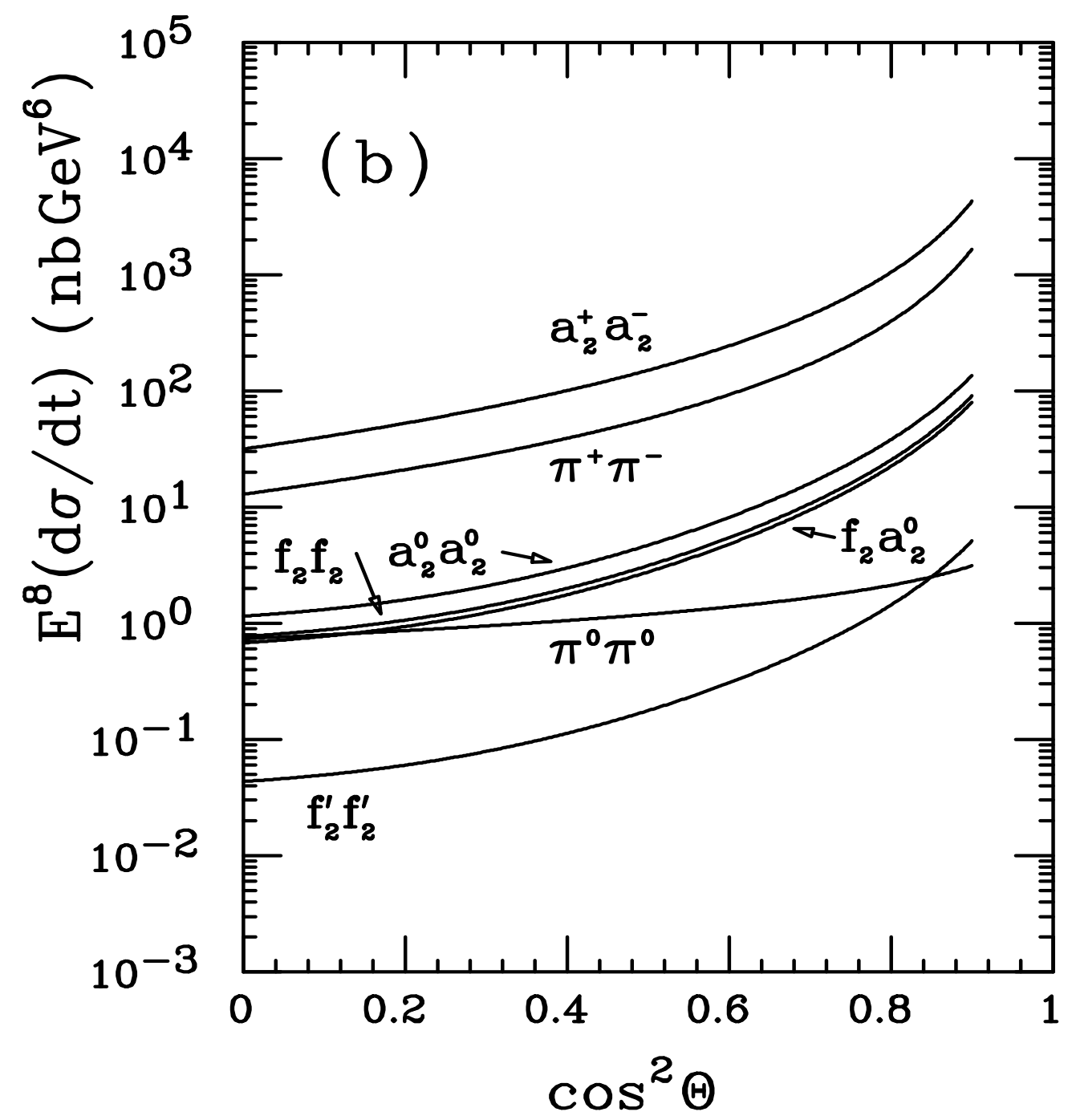

FIG. 2(b) 


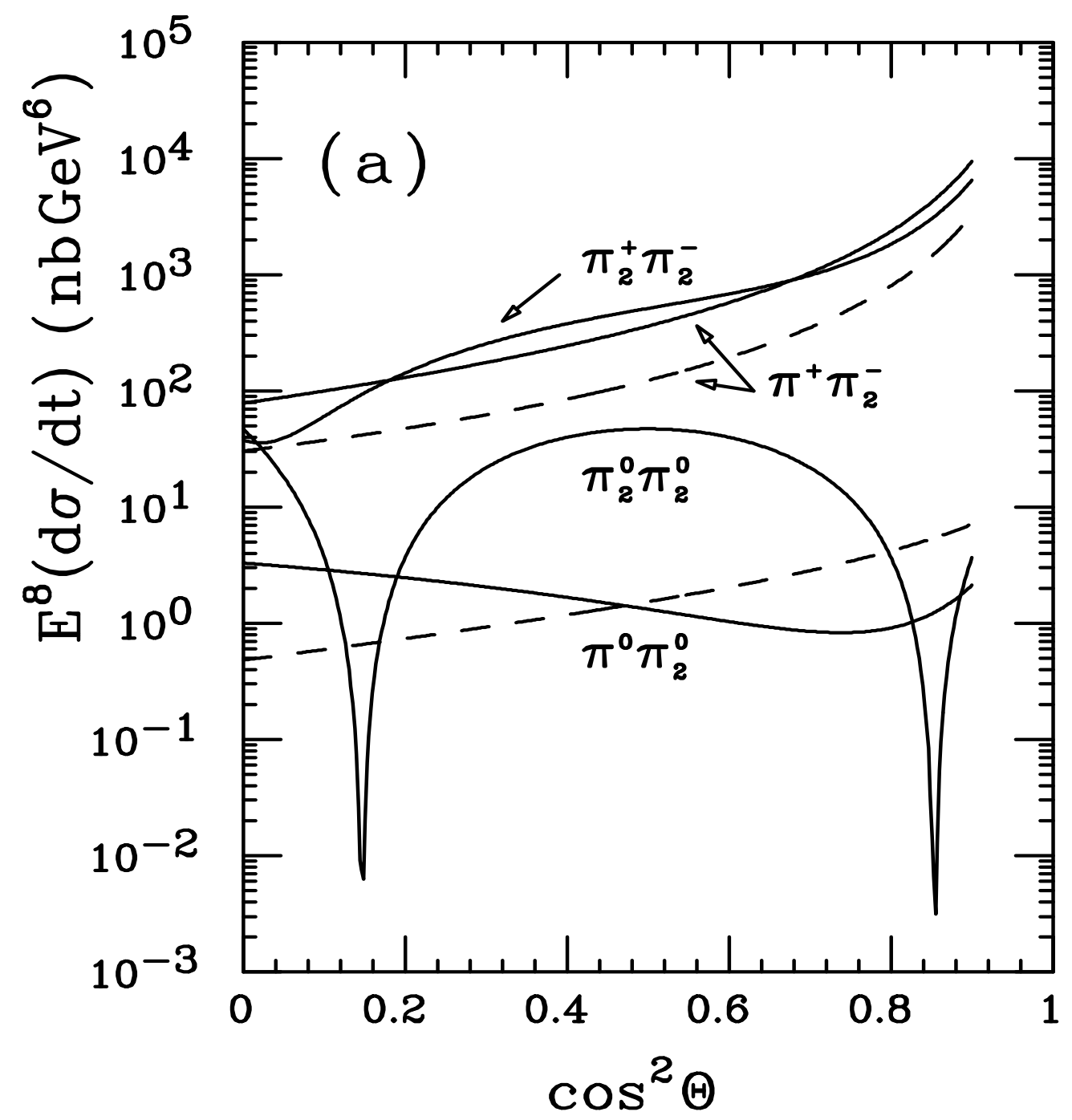

FIG. 3(a) 


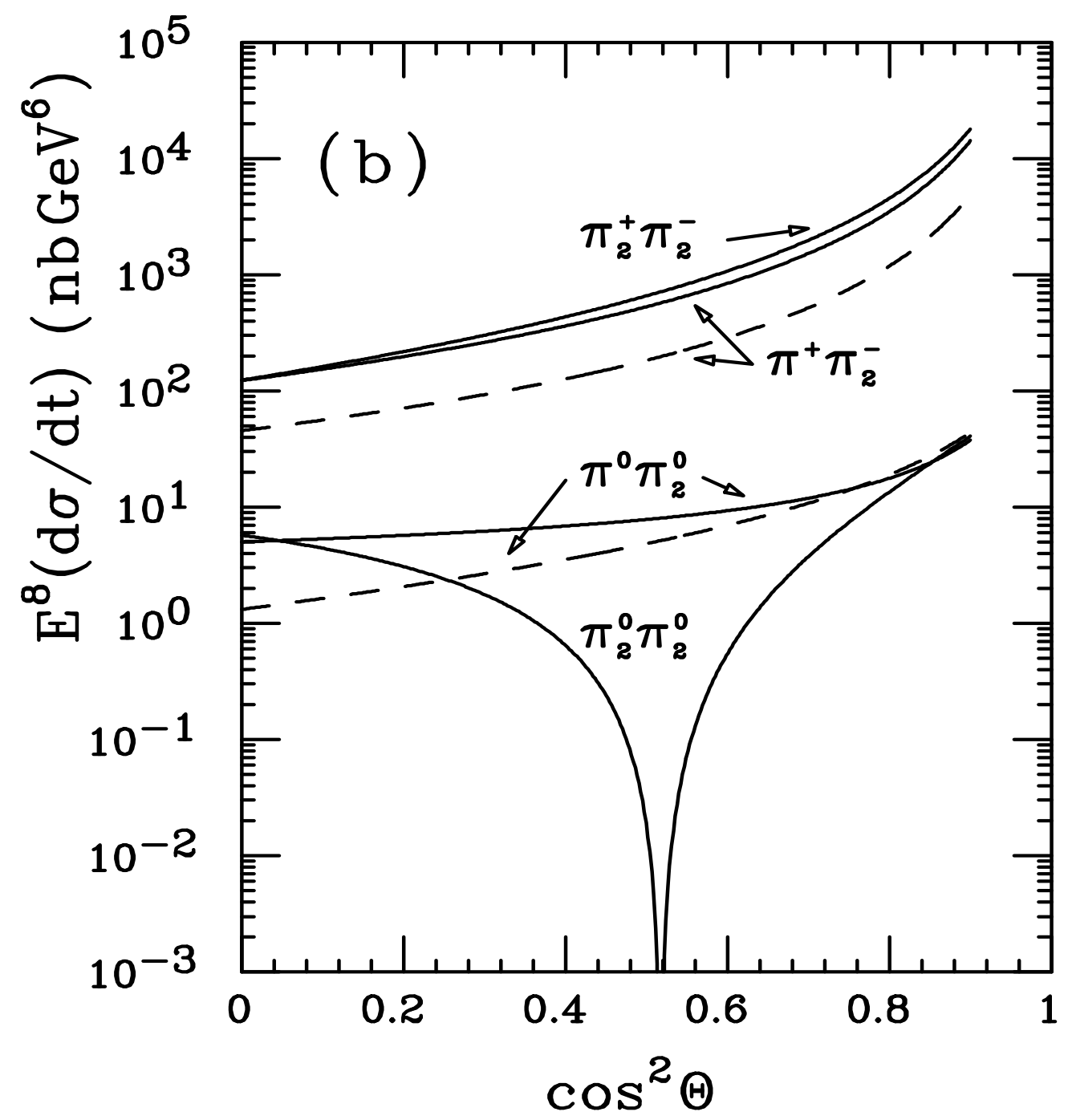

FIG. 3(b) 


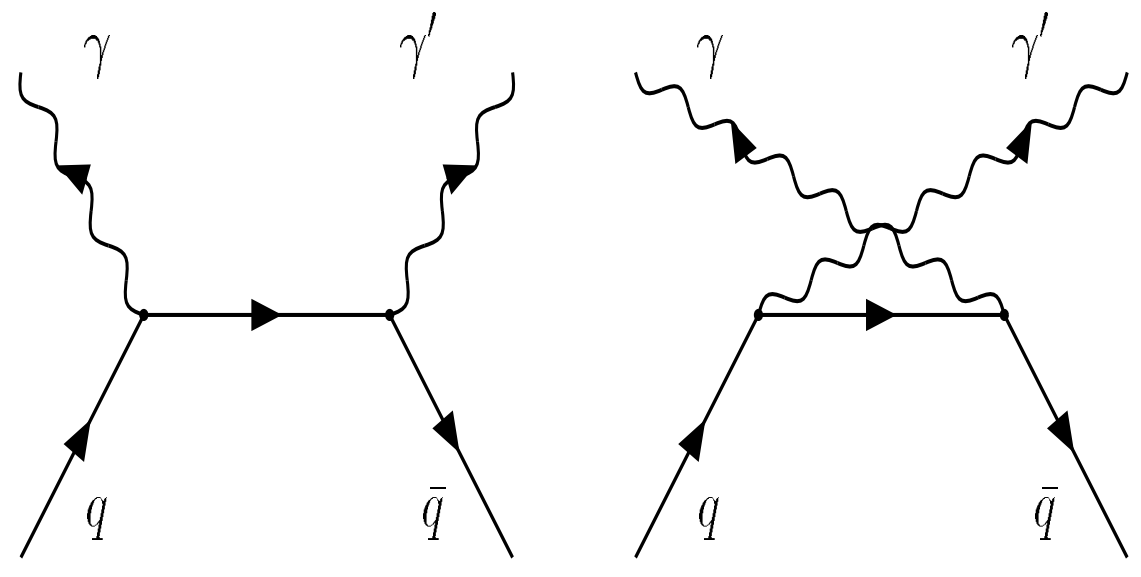

FIG. 4 


\begin{tabular}{ccc}
\hline \hline \multirow{2}{*}{$Q Q^{\prime}$} & \multicolumn{2}{c}{$\sigma\left(e e^{\prime} \rightarrow e e^{\prime} Q Q^{\prime}\right)\left[10^{-40} \mathrm{~cm}^{2}\right]$} \\
\cline { 2 - 3 } & $\mathrm{NR}$ & $\mathrm{GASY}$ \\
\hline$f_{2} f_{2}$ & 20.9 & 25.4 \\
$a_{2}^{0} a_{2}^{0}$ & 30.8 & 53.0 \\
$f_{2} a_{2}^{0}$ & 19.4 & 33.2 \\
$f_{2}^{\prime} f_{2}^{\prime}$ & 0.6 & 1.1 \\
$a_{2}^{+} a_{2}^{-}$ & 291.0 & 1003.3 \\
$\pi_{2}^{0} \pi_{2}^{0}$ & 141.1 & 23.2 \\
$\pi_{2}^{+} \pi_{2}^{-}$ & 749.3 & 1418.2 \\
$\pi_{\mathrm{CZ}}^{0} \pi_{2}^{0}$ & 116.6 & 263.0 \\
$\pi_{\mathrm{ASY}}^{0} \pi_{2}^{0}$ & 35.2 & 104.1 \\
$\pi_{\mathrm{CZ}}^{+} \pi_{2}^{-}$ & 4167.0 & 6562.6 \\
$\pi_{\mathrm{ASY}}^{+} \pi_{2}^{-}$ & 1495.8 & 2368.5 \\
\hline \hline
\end{tabular}

Table 1

\begin{tabular}{ccc}
\hline \hline \multirow{2}{*}{$Q Q^{\prime}$} & \multicolumn{2}{c}{$\sigma\left(e e^{\prime} \rightarrow e e^{\prime} Q Q^{\prime}\right)\left[10^{-40} \mathrm{~cm}^{2}\right]$} \\
\cline { 2 - 3 } & $\mathrm{NR}$ & $\mathrm{GASY}$ \\
\hline$f_{2} f_{2}$ & 1.0 & 0.9 \\
$a_{2}^{0} a_{2}^{0}$ & 1.9 & 2.8 \\
$f_{2} a_{2}^{0}$ & 1.2 & 1.7 \\
$f_{2}^{\prime} f_{2}^{\prime}$ & 0.05 & 0.08 \\
$a_{2}^{+} a_{2}^{-}$ & 16.4 & 55.6 \\
$\pi_{2}^{0} \pi_{2}^{0}$ & 17.5 & 3.4 \\
$\pi_{2}^{+} \pi_{2}^{-}$ & 72.3 & 126.2 \\
$\pi_{\mathrm{CZ}}^{0} \pi_{2}^{0}$ & 5.7 & 11.5 \\
$\pi_{\mathrm{ASY}}^{0} \pi_{2}^{0}$ & 1.4 & 4.1 \\
$\pi_{\mathrm{CZ}}^{+} \pi_{2}^{-}$ & 156.7 & 246.5 \\
$\pi_{\mathrm{ASY}}^{+} \pi_{2}^{-}$ & 56.0 & 88.7 \\
\hline \hline
\end{tabular}

Table 2 


\begin{tabular}{ccc}
\hline \hline \multirow{2}{*}{$Q Q^{\prime}$} & \multicolumn{2}{c}{$\sigma\left(e e^{\prime} \rightarrow e e^{\prime} Q Q^{\prime}\right)\left[10^{-40} \mathrm{~cm}^{2}\right]$} \\
\cline { 2 - 3 } & $\mathrm{NR}$ & $\mathrm{GASY}$ \\
\hline$f_{2} f_{2}$ & 1.4 & 1.4 \\
$a_{2}^{0} a_{2}^{0}$ & 2.3 & 3.6 \\
$f_{2} a_{2}^{0}$ & 1.5 & 2.3 \\
$f_{2}^{\prime} f_{2}^{\prime}$ & 0.04 & 0.06 \\
$a_{2}^{+} a_{2}^{-}$ & 20.1 & 68.6 \\
$\pi_{2}^{0} \pi_{2}^{0}$ & 10.0 & 1.6 \\
$\pi_{2}^{+} \pi_{2}^{-}$ & 41.6 & 72.4 \\
$\pi_{\mathrm{CZ}}^{0} \pi_{2}^{0}$ & 12.0 & 24.5 \\
$\pi_{\mathrm{ASY}}^{0} \pi_{2}^{0}$ & 3.0 & 8.8 \\
$\pi_{\mathrm{CZ}}^{+} \pi_{2}^{-}$ & 341.1 & 536.7 \\
$\pi_{\mathrm{ASY}}^{+} \pi_{2}^{-}$ & 122.0 & 193.2 \\
\hline \hline
\end{tabular}

Table 3

\begin{tabular}{ccc}
\hline \hline$Q Q^{\prime}$ & $\left\langle\left(e_{1}-e_{2}\right)^{2}\right\rangle$ & $\left\langle e_{1} e_{2}\right\rangle$ \\
\hline$f_{2} f_{2}$ & 0 & $5 / 18$ \\
$a_{2}^{0} a_{2}^{0}$ & 0 & $5 / 18$ \\
$f_{2} a_{2}^{0}$ & 0 & $1 / 6$ \\
$f_{2}^{\prime} f_{2}^{\prime}$ & 0 & $1 / 9$ \\
$a_{2}^{+} a_{2}^{-}$ & 1 & $-2 / 9$ \\
$\pi_{2}^{0} \pi_{2}^{0}$ & 0 & $5 / 18$ \\
$\pi_{2}^{+} \pi_{2}^{-}$ & 1 & $-2 / 9$ \\
$\pi^{0} \pi_{2}^{0}$ & 0 & $5 / 18$ \\
$\pi^{+} \pi_{2}^{-}$ & 1 & $-2 / 9$ \\
\hline \hline
\end{tabular}

Table B1 


\begin{tabular}{cccc}
\hline \hline \multirow{2}{*}{$Q$} & $\Gamma(Q \rightarrow \gamma \gamma)(\mathrm{keV})$ & \multicolumn{2}{c}{$\left|f_{L}\right|(\mathrm{MeV})$} \\
\cline { 3 - 4 } & & $\mathrm{NR}$ & $\mathrm{GASY}$ \\
\hline$f_{2}(1270)$ & 2.440 & 119 & 88 \\
$a_{2}(1320)$ & 1.040 & 131 & 97 \\
$f_{2}^{\prime}(1525)$ & 0.097 & 92 & 68 \\
$\pi_{2}(1670)$ & 1.350 & 845 & 290 \\
\hline \hline & & & \\
\hline & Table C1
\end{tabular}

